\title{
High Precision 3D Single Molecule Microscopy Using the Double Helix Point Spread Function
}

Anurag Agrawal, Warren Colomb and Scott Gaumer

Double Helix Optics, Inc., Boulder, Colorado, United States

Optical diffraction limits resolution in visible spectrum to $200 \mathrm{~nm}$ in the lateral dimension (x-y) and 500 $\mathrm{nm}$ in axial dimension $(\mathrm{z})$. Recent advances in engineering properties of fluorescent proteins and dyes have enabled nanometer scale visualization by localizing sparse ensembles of photoswitchable/photoactivatable molecules through many frames. A final image is formed by combining locations of all the molecules to form a "super-resolution image". The family of techniques is known as single-molecule localization microscopy (SMLM)[1]. Although SMLM enables high precision imaging of 10-20 nm in the lateral dimension, it lacks axial (z) resolution, especially near focus. One method to extract axial information uses an astigmatic lens to distort the point spread function, thereby enabling extraction of a limited amount of 3D information. Unfortunately, this approach has a limited depth capability of only about 500-700 nm.

The Double-Helix Point Spread function (DH-PSF) offers a solution to this problem by enabling highdepth and high-precision 3D imaging. The DH-PSF refers to an optical response that modifies the point spread function on the microscope such that, instead of an Airy Disc, the image of each point source is in the form of two well separated lobes. Unlike the blurring of the Airy Disc, the two lobes rotate around their midpoint as the emitter moves along the axial dimension. The axial dimension of the emitter is therefore encoded in the angle of the two lobes and the center point between them indicates the lateral position [2]. The DH-PSF technique has been shown to be fundamentally more precise than other 3D methods, providing the best resolution and the longest depth of field [3]. This unique combination of precision and depth can be used for 3D-SMLM, 3D-particle tracking, and widefield restoration microscopy.

The DH-PSF along with other engineered point spread function designs, collectively known as E-PSFs (Tetrapod [4], Single Helix, etc.) enable optimal control of the PSF based on parameters such as desired depth-range, emission spectrum, and signal-to-noise ratio, etc. An important advantage of these PSFs is the increase in depth of field by a factor of 2-30x with respect to a conventional microscope of the same numerical aperture (NA).

The E-PSF response is implemented by inserting a special optical element (also known as a phase mask) in the pupil plane of the imaging system. However, for most microscopy systems, the pupil plane lies inside the microscope objective. An implementation of this technique is currently available in the form of an optical module - the SPINDLE ${ }^{\circledR}$ [5], [6]- that can be easily integrated with most scientific microscopes and cameras (see Figure 1). The SPINDLE provides easy access to the pupil plane such that the phase mask can be easily inserted and aligned. Furthermore, the SPINDLE ${ }^{2}$ system, allows for imaging of two image channels (separated by emission spectrum and/or polarization) allowing independent modification of the PSF of each channel using the library of phase masks.

The images captured using the SPINDLE and E-PSF technology are processed with the Double Helix 3DTRAX ${ }^{\mathrm{TM}}$ software. The software uses a calibration stack to relate the shape (rotation) of the PSF to the axial position, and even color, of the emitter and localizes the $3 \mathrm{D}$ position of each molecule. The resulting localizations can then be drift corrected for visualizing the data or processed through the 3D particle tracking algorithm. The data can be exported in an open format for further analysis. 
Figure 2 shows a DH-PSF reconstruction of microtubules in a mouse embryonic fibroblast (MEF). The DH-PSF super-resolution reconstruction captured a depth of $2.5 \mu \mathrm{m}$ with a lateral precision of $<20 \mathrm{~nm}$ and axial precision of $<30 \mathrm{~nm}$. When compared to the 2D widefield image, the DH-PSF 3D images show precise reconstructions of microtubules that were otherwise lost in the background (see Figure 2 bottom images). Moreover, the extended depth of field of the DH-PSF captures additional localizations deeper into the sample allowing a more complete reconstruction of the sample. The unique combination of multimodal image with engineered PSFs has been used to extend the imaging capabilities of microscopes for nanometer-scale single-molecule imaging[7], [8], light sheet[9], 3D particle tracking [4], [10], in the study of cancer[11], immunology, neuroscience[5], and more.

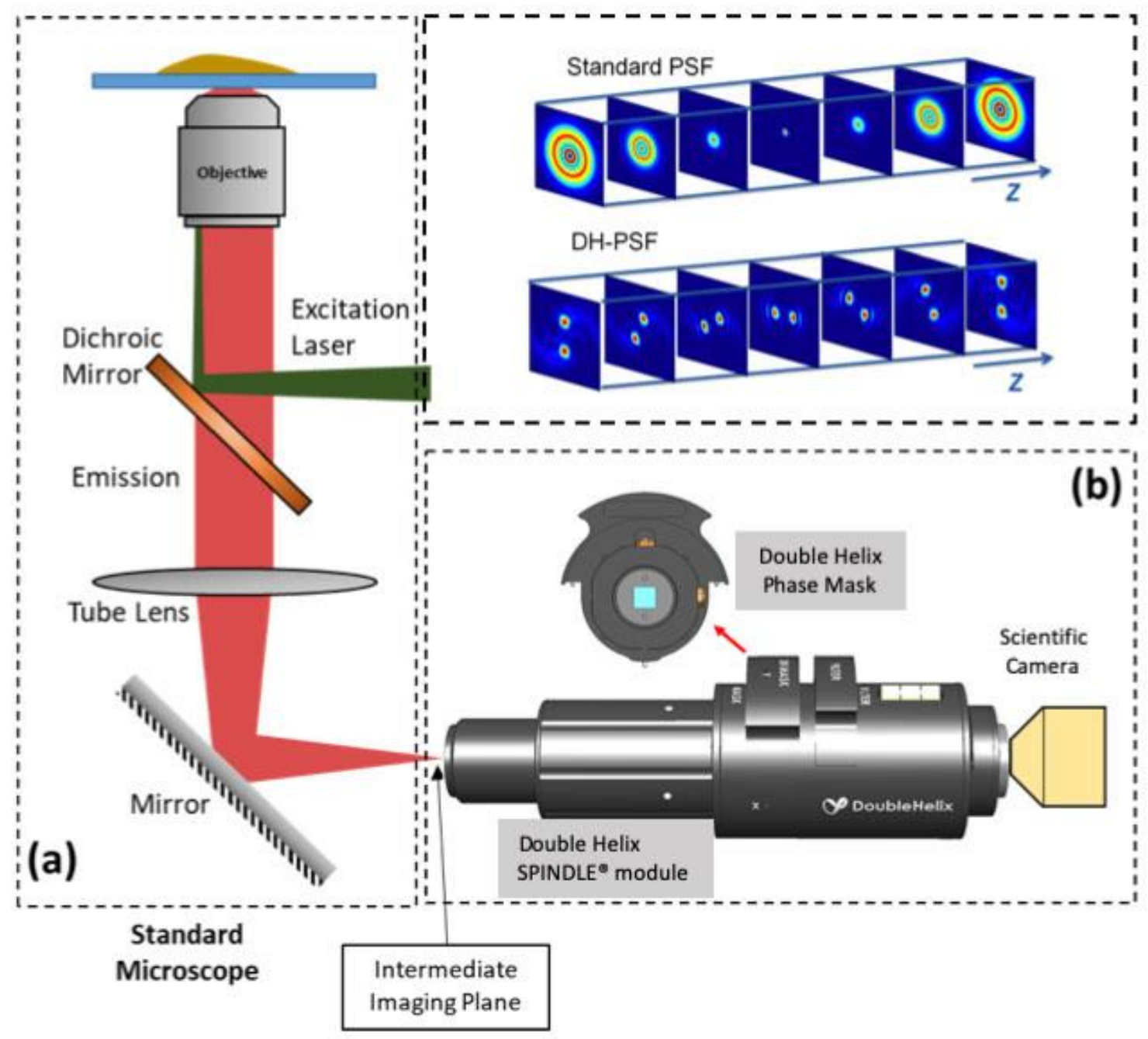

Figure 1. The PSF of a Standard Microscope (a) is modified by inserting a Double-Helix phase mask in the SPINDLE module (b) where the pupil plane of the objective is imaged. (c) Unlike the standard PSF, the DH-PSF exhibits two lobes that are oriented at different angles based on the axial location of the object. 

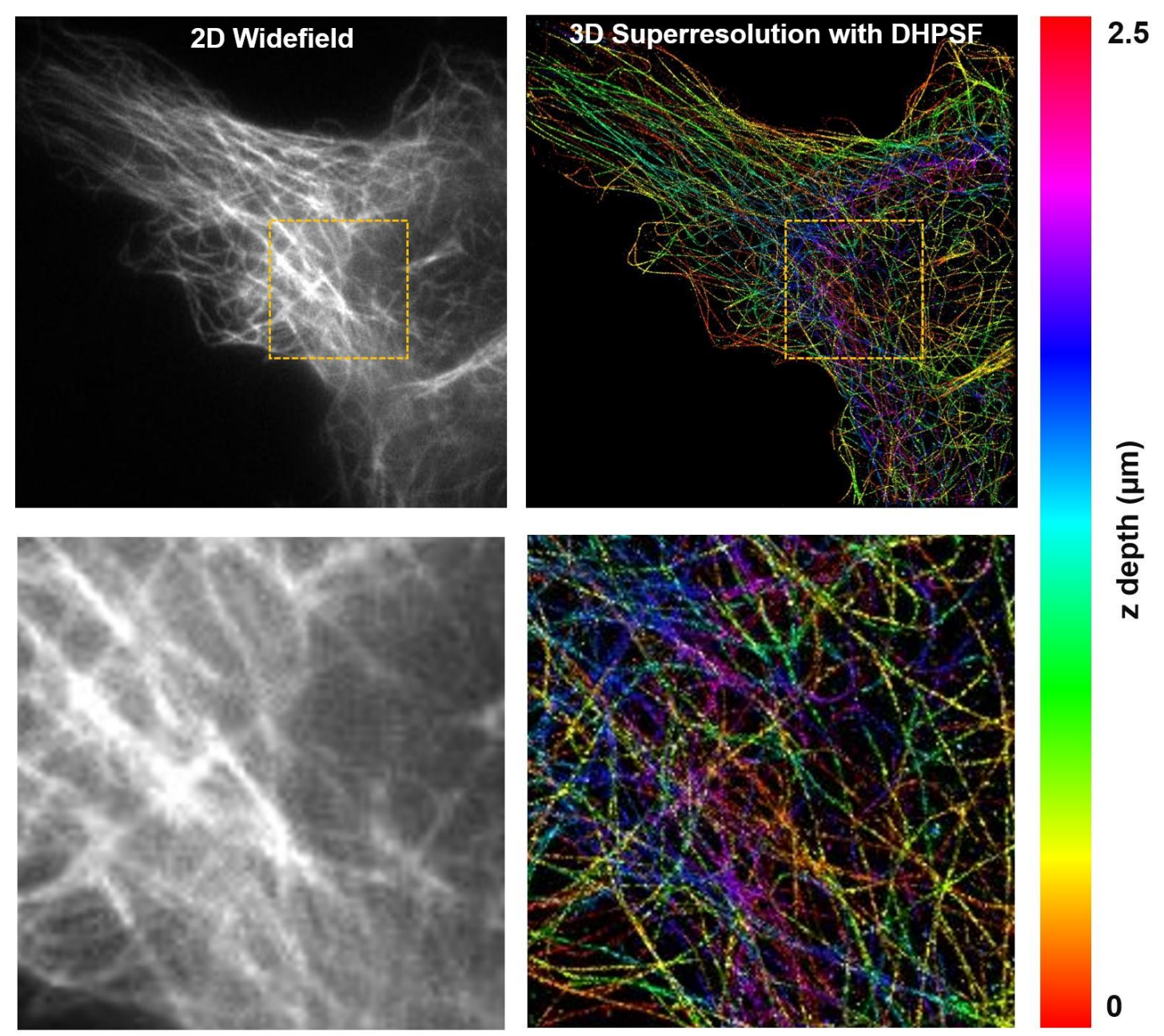

\section{5}

Figure 2. 3D DHPSF super-Resolution Image (right) of microtubules captures the detailed 3D information not seen in conventional 2D widefield imaging (left). The bottom row shows magnified insets illustrating the image detail further. The $\mathrm{z}$ depth is encoded in color (scale on the right)

\section{References}

[1] QM. J. Rust, et. al., "Sub-diffraction-limit imaging by stochastic optical reconstruction microscopy (STORM).," Nat. Methods, vol. 3, no. 10, pp. 793-5, Oct. 2006.

[2] S. R. P. Pavani et. al., "High-efficiency rotating point spread functions," Opt. Express, vol. 16, no. 5, pp. 3484-3489, Mar. 2008.

[3] G. Grover, et. al., "Performance limits on three-dimensional particle localization in photon-limited microscopy," Opt. Lett., vol. 35, no. 19, p. 3306, Sep. 2010.

[4] Y. Shechtman, et. al., "Precise Three-Dimensional Scan-Free Multiple-Particle Tracking over Large Axial Ranges with Tetrapod Point Spread Functions," Nano Lett., vol. 15, no. 6, pp. 4194-4199, Jun. 2015.

[5] S. Jain, et. al., "ATPase-Modulated Stress Granules Contain a Diverse Proteome and Substructure," Cell, vol. 164, no. 3, pp. 487-498, 2016.

[6] D. Wang, et. al., "Enhanced information content for three-dimensional localization and tracking using the double-helix point spread function with variable-angle illumination epifluorescence microscopy," Appl. Phys. Lett., vol. 110, no. 21, p. 211107, May 2017. 
[7] A. Gahlmann et al., "Quantitative multicolor subdiffraction imaging of bacterial protein ultrastructures in three dimensions.," Nano Lett., vol. 13, no. 3, pp. 987-93, Mar. 2013.

[8] S. R. P. Pavani et al., "Three-dimensional, single-molecule fluorescence imaging beyond the diffraction limit by using a double-helix point spread function.," Proc. Natl. Acad. Sci. U. S. A., vol. 106, no. 9, pp. 2995-9, Mar. 2009.

[9] A.-K. Gustavsson, et. al., "3D single-molecule super-resolution microscopy with a tilted light sheet," Nat. Commun. 2018 91, vol. 9, no. 1, p. 123, Jan. 2018.

[10] Y. Shechtman, et. al., "Multicolour localization microscopy by point-spread-function engineering," Nat. Photonics, vol. 10, no. 9, pp. 590-594, Sep. 2016.

[11] W. Y. Lam et al., "HER2 Cancer Protrusion Growth Signaling Regulated by Unhindered, Localized Filopodial Dynamics," bioRxiv, p. 654988, Jun. 2019. 